\title{
Improved Suzuki-Miyaura reaction conversion efficiency using magnetic nanoparticles and inductive heating
}

\author{
Alejandro Villacampa ${ }^{1}$, Luis Duque ${ }^{1}$, Olga Juanes ${ }^{2}$, Francisco Javier Palomares ${ }^{3}$, \\ Pilar Herrasti ${ }^{1}$, and Nieves Menéndez ${ }^{1, *}$ (i) \\ ${ }^{1}$ Departamento de Química Física Aplicada, Facultad de Ciencias, Universidad Autónoma de Madrid, Cantoblanco, Madrid, Spain \\ ${ }^{2}$ Departamento de Química Orgánica, Facultad de Ciencias, Universidad Autónoma de Madrid, Cantoblanco, Madrid, Spain \\ ${ }^{3}$ Instituto de Ciencia de Materiales de Madrid (ICMM), Consejo Superior de Investigaciones Científicas (CSIC), Cantoblanco, Madrid, \\ Spain
}

Received: 6 May 2021

Accepted: 25 September 2021

Published online:

3 January 2022

(C) The Author(s) 2021

\begin{abstract}
The use of magnetic nanoparticles in $\mathrm{C}-\mathrm{C}$ coupling reactions enables the facile recovery of the catalyst under environmentally friendly conditions. Herein, the synthesis of $\mathrm{Pd} / \mathrm{Fe} @ \mathrm{Fe}_{3} \mathrm{O}_{4}$ nanoparticles by the reduction of $\mathrm{Pd}^{2+}$ and oxidation of $\mathrm{Fe}$ on the surface of preformed $\mathrm{Fe}_{\mathrm{Fe}} \mathrm{Fe}_{3}$ is reported. The nanoparticles were characterized using a variety of analytical techniques (transmission electron microscopy, Mössbauer spectroscopy, X-ray photoelectron spectroscopy, and $X$-ray diffraction) to determine their size, structure, and chemical composition. The catalytic efficiency of these nanoparticles in classical Suzuki-Miyaura coupling reactions was investigated. The nanoparticles achieved high catalytic activity with the application of local heating by an alternating magnetic field. An investigation was conducted at identical temperatures to compare global heating with the application of an external magnetic field; magnetic heating demonstrated excellent substrate conversion in lesser time and at a lower temperature. The catalyst could also be recycled and reused three times, with $\sim 30 \%$ decrease in the substrate conversion, which is most likely due to the agglomeration of the Pd nanoparticles or poisoning of the Pd catalyst. This approach, which takes advantage of the catalytic activity and magnetic susceptibility of magnetic nanoparticles, can be applied to several organic transformations to improve their efficiency.
\end{abstract}

Handling Editor: Dale Huber.

Address correspondence to E-mail: nieves.menendez@uam.es 


\section{GRAPHICAL ABSTRACT}

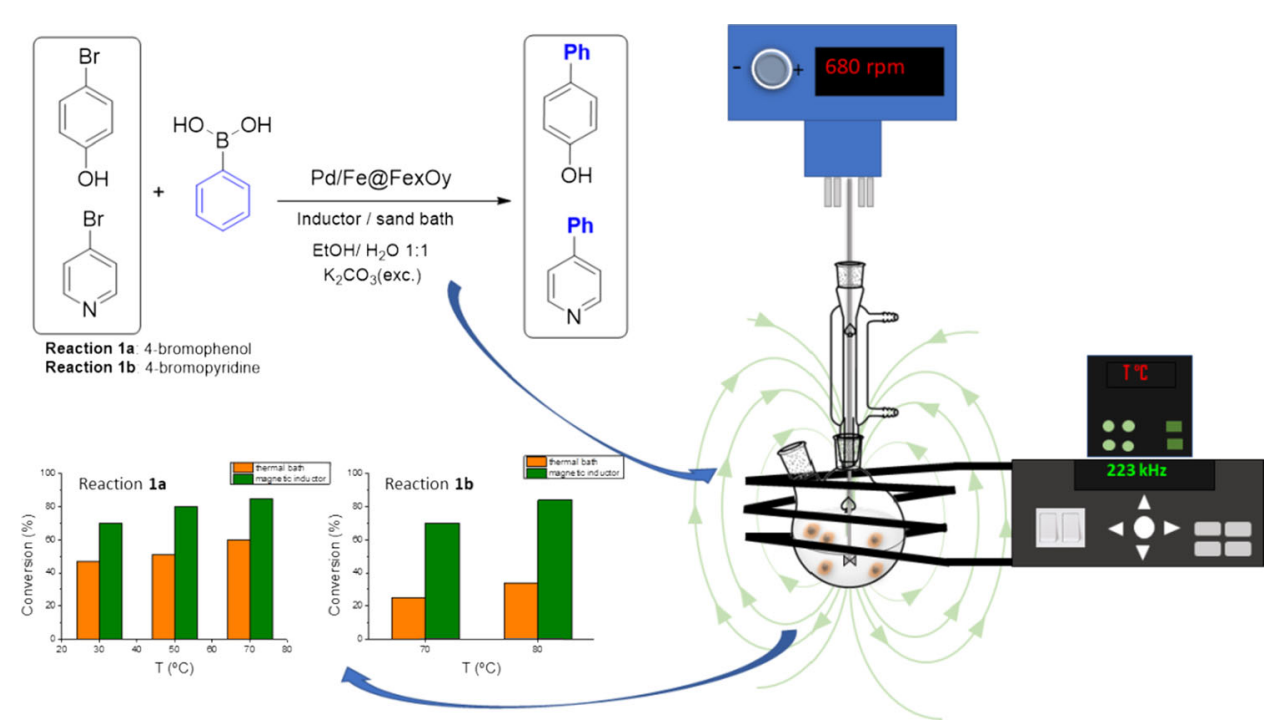

\section{Introduction}

The use of transition metal compounds containing elements such as $\mathrm{Pd}, \mathrm{Au}, \mathrm{Ni}, \mathrm{Cu}$, and $\mathrm{Ru}$ to catalyze different organic reactions is well known [1-7]. The Suzuki-Miyaura reaction, a reaction between a halogenated hydrocarbon and an arylboronic acid, was first reported in 1979 [8] and is widely used as a route to synthesize $\mathrm{C}-\mathrm{C}$ bonds. It is mainly catalyzed by Pd owing to its high activity and selectivity and is one of the most widely accepted methods for the synthesis of pharmaceuticals [9]. The advantages of the Suzuki-Miyaura reaction include tolerance to aqueous media, functional group diversity and tolerance, high conversions, and mild reaction conditions.

Traditional reactions using homogeneous Pd complexes as catalysts have been described [10, 11]. The complexes exhibited high performance and stereoselectivity, but the formation of insoluble Pd black or aggregation of the insoluble species diminished its catalytic activity in the coupling reaction [12, 13]. In addition, it is very difficult to recycle and recover homogeneous catalysts as they remain in the reaction medium at low concentrations and cannot be separated from the synthesis product, which increases the cost of the process and potentially results in catalyst unavailability and product impurity [14]. Therefore, it is necessary to design economical and efficient environmental processes for the separation and reuse of catalysts. This approach has attracted enormous attention in the twenty-first century.

Using a heterogeneous catalyst can efficiently solve these problems and facilitate easy separation of the catalyst from the reaction mixture [15]. Pd has been immobilized on silica nanoparticles [16, 17], graphene [18], carbon nanotubes [19], metal-organic frameworks [20, 21], and metal oxides [22, 23]; however, these catalysts are expensive and usually require complicated and tedious preparation procedures. To overcome these drawbacks, the development of practical, economical, and environmentally friendly supported Pd catalysts is required.

The anchoring of catalysts to magnetic nanoparticles (MNPs) has been extensively studied in the recent years [24-26]. Iron oxide nanoparticles exhibit many advantages, including a high surface-to-volume ratio and facile magnetic recovery [27]. The magnetically recoverable catalyst has attracted significant attention because its recovery process is more economical and greener compared to centrifugation and filtration. Magnetite is one of the most commonly used metal oxide nanoparticles owing to its magnetic 
properties and biocompatibility. Pd-functionalized magnetite nanoparticles have been reported for application in the Suzuki-Miyaura reaction $[16,22,28]$. In general, the functionalization process involves difficult and time-consuming ligand synthesis and additional costs. To minimize the use of ligands in the $\mathrm{Pd}$ incorporation process, facile procedures based on sequestering processes were studied on materials capable of reducing Pd by oxidizing the surface of the sequestering material. $\mathrm{Li}$ et al. studied the sequestration of $\mathrm{Ni}(\mathrm{II})$ in a solution using Fe nanoparticles [29]. The process involved coordination of the metal ion to the surface of the reactive $\mathrm{Fe}$, which acts as a reductant toward the less reactive metal ions. Applying this concept, $\mathrm{Fe}_{\mathrm{x}} \mathrm{O}_{\mathrm{y}} @ \mathrm{Fe}$ was synthesized to recover a variety of ions, such as $\mathrm{Co}^{2+}$, $\mathrm{Cu}^{2+}, \mathrm{Ni}^{2+}, \mathrm{Rh}^{\mathrm{x}}, \mathrm{Pd}^{2+}, \mathrm{Ag}^{+}$, and $\mathrm{Pt}^{4+}$, from solution $[30,31]$.

The use of heterogeneous catalysts in the SuzukiMiyaura reaction requires relatively elevated reaction temperatures, which can be achieved using a local heating procedure. The magnetic properties of magnetite nanoparticles enable the conversion of electromagnetic induction (EMI) into heat. The use of induction heating offers remarkable benefits compared with other heating methods, such as microwave irradiation and thermal baths, owing to the penetration power of the magnetic field [32]. In addition, EMI can quickly and directly induce high temperatures in catalyst particles [33, 34]. Thus, heating the system by EMI takes less time compared with external heating methods that involve a conventional heat source, such as a thermal bath, and provides greater control and safety over the reaction. The application of PEG- or oleic-acid-coated Fe@ $\mathrm{Fe}_{3-}$ $\mathrm{O}_{4}$ nanoparticles with $\mathrm{Pd}$ adsorbed on the surface in Suzuki-Miyaura and Sonogashira reactions has been reported recently, demonstrating the feasibility of this approach [35]. Our study demonstrates the novel use of uncoated $\mathrm{Pd} / \mathrm{Fe} @ \mathrm{Fe}_{3} \mathrm{O}_{4}$ nanoparticles with excellent catalytic activity in the Suzuki-Miyaura reaction heating by EMI. Nanoparticles are usually coated to prevent agglomeration and loss of their electrical and magnetic properties; however, we show that vigorous agitation does not result in a loss of properties, making it possible to apply lower frequency magnetic fields than those required for coated nanoparticles. The effect of different heat sources and temperatures on the efficiency of the heterogeneous catalytic Suzuki-Miyaura reaction was analyzed, and the results indicated that inductive heating improved the catalyst efficiency. The recovery and reuse of the catalysts was also tested.

\section{Experimental section}

\section{Catalyst synthesis}

The catalyst was prepared in two stages according to a previously reported procedure [31, 36]. Briefly, a solution of $\mathrm{NaBH}_{4}(2.24 \mathrm{~g}, 59 \mathrm{mmol})$ in $30 \mathrm{~mL}$ of deionized (DI) water was added dropwise (for a period of approximately $15 \mathrm{~min}$ ) to a solution of $\mathrm{FeCl}_{3} \cdot 6 \mathrm{H}_{2} \mathrm{O}(5.67 \mathrm{~g}, 21 \mathrm{mmol})$ in DI water $(400 \mathrm{~mL})$. The mixture was mechanically stirred at $600 \mathrm{rpm}$ for $4 \mathrm{~h}$, to form a black precipitate. The nanoparticles were separated using a magnet, and the supernatant was discarded. The solid was washed and sonicated with DI water three times, and finally with ethanol to produce a transparent solution. Finally, the resulting black paste was centrifuged and vacuum-dried overnight at $25^{\circ} \mathrm{C}$ to yield $\mathrm{Fe} @ \mathrm{Fe}_{\mathrm{x}} \mathrm{O}_{\mathrm{y}}$ powder $(1 \mathrm{~g})$. In the second stage, to obtain the $\mathrm{Pd} / \mathrm{Fe} @ \mathrm{Fe}_{\mathrm{x}} \mathrm{O}_{\mathrm{y}}$ catalyst, $200 \mathrm{mg}$ of $\mathrm{Fe} @ \mathrm{Fe}_{\mathrm{x}} \mathrm{O}_{\mathrm{y}}$ was reacted with $\mathrm{Pd}\left(\mathrm{NO}_{3}\right)_{2}$ (230 mg, $1 \mathrm{mmol}$ ) of in $250 \mathrm{~mL}$ of DI water at a pH of 10.5 under an argon atmosphere. The mixture was sonicated for $4 \mathrm{~h}$ increasing the temperature to $45-50$ ${ }^{\circ} \mathrm{C}$. The reaction mixture was subsequently centrifuged for $10 \mathrm{~min}$ at $10000 \mathrm{rpm}$, and the black precipitate was washed with distilled water several times, followed by vacuum drying for $12 \mathrm{~h}$ at $25^{\circ} \mathrm{C}$. The synthesis and purification steps were carried out in air, although the catalyst was stored in an inert atmosphere to prevent oxidation over time.

\section{Material characterization}

Phase identification analysis of the MNPs was conducted by X-ray diffraction (XRD) using a Bruker D8 powder diffractometer equipped with a primary monochromator and an ultrafast Lynxeye XE-T multichannel detector with $\mathrm{Cu} K \alpha_{1}$ radiation. The patterns were collected in the $2 \theta$ range of $5-80^{\circ}$, with an angular increment of $0.02^{\circ}$ and an increment time of $1 \mathrm{~s}$. The diffractograms were analyzed using the PANalytical X'Pert HighScore program, and their profile fitting was carried out to obtain more reliable peak parameters after deconvolution of the overlapped peaks. Peaks were assigned based on the 
International Center for Diffraction Data (ICDD) reference considering the peak positions and intensities. A semiquantitative XRD analysis of the composition based on the reference intensity ratio (RIR) was also performed. The morphology of the catalyst was studied using a transmission electron microscope (JEOL JEM 1010) operated at $100 \mathrm{kV}$. The samples were prepared by placing a single droplet $(10 \mu \mathrm{L})$ of an aqueous solution of MNPs onto a copper grid coated with a carbon film. The grid was dried in air for several hours at room temperature (RT). Mössbauer spectroscopy was used to analyze the Fe phases in the samples. The spectra were recorded at RT in the triangular mode using a transmission spectrometer with a ${ }^{57} \mathrm{Co} / \mathrm{Rh}$ source. Spectral analyses were performed by nonlinear fitting using the NORMOS program [37]. Each spectrum was fitted by the convolution of subspectra corresponding to as many different crystallographic positions of $\mathrm{Fe}$ that could be identified in the material by XRD. For each Fe site, the isomer shift ( $\delta)$, quadrupole splitting $\left(\Delta \mathrm{E}_{\mathrm{Q}}\right)$, and hyperfine magnetic field $(\mathrm{H})$ were fitted. We fixed equal full width at half maximum of the Lorentzian lines of each component, and the intensity ratio 3:2:1:1:2:3 for each sextet. The combination of these three parameters allows for the identification of a particular compound. The energy calibrations were performed using an $\alpha-\mathrm{Fe}(6 \mu \mathrm{m})$ foil. The composition of the material was also examined by inductively coupled plasma optical emission spectroscopy (ICPOES) using a Perkin-Elmer Optima 2100 DV system to determine the Pd loading on the catalyst before and after its use and the possible leaching of Fe and/ or Pd; each sample was analyzed three times. X-ray photoelectron spectroscopy (XPS) was used to characterize the surface chemistry of the samples and the oxidation states of Fe and Pd. XPS experiments were performed in a UHV chamber with a base pressure of $10^{-10}$ mbar equipped with a hemispherical electron energy analyzer (SPECS Phoibos 150 spectrometer) and a 2D delay-line detector (Surface Concept), using a non-monochromatic $\mathrm{X}$-ray source of $\mathrm{Al} \mathrm{K} \alpha$ radiation $(1486.6 \mathrm{eV})$ operated at $300 \mathrm{~W}$. XPS spectra were recorded at the normal emission take-off angle, using an energy step of 0.50 and $0.10 \mathrm{eV}$ and a pass-energy of 40 and $20 \mathrm{eV}$ for survey spectra and detailed corelevel regions (Fe 2p, Pd 3d, C 1 s, Fe 3p, Pd 4p, and O $1 \mathrm{~s})$, respectively. The charging effects accumulated in the photoemission process were compensated using a low energy electron flood gun, in which a wide beam illuminates the sample surface during the measurements. On the one hand, this allows the binding energy calibration to be referenced to the $\mathrm{C}$ $1 \mathrm{~s}$ photoelectron peak at $284.5 \mathrm{eV}$, which was checked before and after the measurement of every selected core-level transition. On the other hand, and more importantly, charge compensation also provides the correct lineshape of a given core-level emission, which is significantly affected in the absence of flood-gun irradiation and unfortunately leads to contradictory conclusions in the determination of the oxidation states present. Data processing was performed using CasaXPS software (Casa Software Ltd., Cheshire, UK). Spectra are displayed after the subtraction of the contribution of the Al-Ka satellite emission; in some cases, the spectra were normalized to the maximum intensity to highlight the lineshape differences, which provides direct valuable insight into the oxidation states. ${ }^{1} \mathrm{H}-\mathrm{NMR}$ spectroscopy was performed on a Bruker Avance $300 \mathrm{MHz}$ instrument to determine the conversion of the Suzuki-Miyaura reactions. To calculate the conversion, each ${ }^{1} \mathrm{H}-\mathrm{NMR}$ spectrum was integrated relative to the internal standard 1,3,5trimethoxybenzene. The samples were prepared by dissolving $0.2 \mathrm{~mL}$ of the crude reaction in $0.4 \mathrm{~mL}$ of deuterated dimethylsulfoxide (DMSO- $d_{6}$ ).

\section{Suzuki-Miyaura coupling reactions}

The efficiency of the $\mathrm{Pd} / \mathrm{Fe} @ \mathrm{Fe}_{3} \mathrm{O}_{4}$ catalyst under magnetic induction heating was tested in the catalyzed coupling of phenylboronic acid with 4-bromophenol and 4-bromopyridine. The conversions of both reactions were compared using two heating sources: a conventional thermal bath and an alternating magnetic field. A schematic of the reactions and the magnetic system is shown in Fig. 1 .

For reaction 1a, 4-bromophenol $(1.8 \mathrm{mmol})$, phenylboronic acid ( $2.7 \mathrm{mmol})$, and 1,3,5-trimetoxybenzene $(1.8 \mathrm{mmol})$ as an internal standard were dissolved in $5 \mathrm{~mL}$ of ethanol. $\mathrm{K}_{2} \mathrm{CO}_{3}(3.6 \mathrm{mmol})$ was dissolved in $5 \mathrm{~mL}$ of $\mathrm{DI}$ water and added to the mixture with $30 \mathrm{mg}$ of $\mathrm{Pd} / \mathrm{Fe} @ \mathrm{Fe}_{\mathrm{x}} \mathrm{O}_{\mathrm{y}}(5 \mathrm{~mol} \% \mathrm{Pd}$ ). The reaction mixtures were mechanically stirred in a glass reactor in air over the required reaction times.

For reaction 1b, 4-bromopyridine hydrochloride (1.8 mmol), phenylboronic acid ( $2.7 \mathrm{mmol})$, and 1,3,5trimetoxybenzene $(1.8 \mathrm{mmol})$ were dissolved in $5 \mathrm{~mL}$ of ethanol. $\mathrm{K}_{2} \mathrm{CO}_{3} \quad(7.2 \mathrm{mmol}$ because 
Figure 1 Schematic of the Suzuki-Miyaura reaction conducted under induction heating.

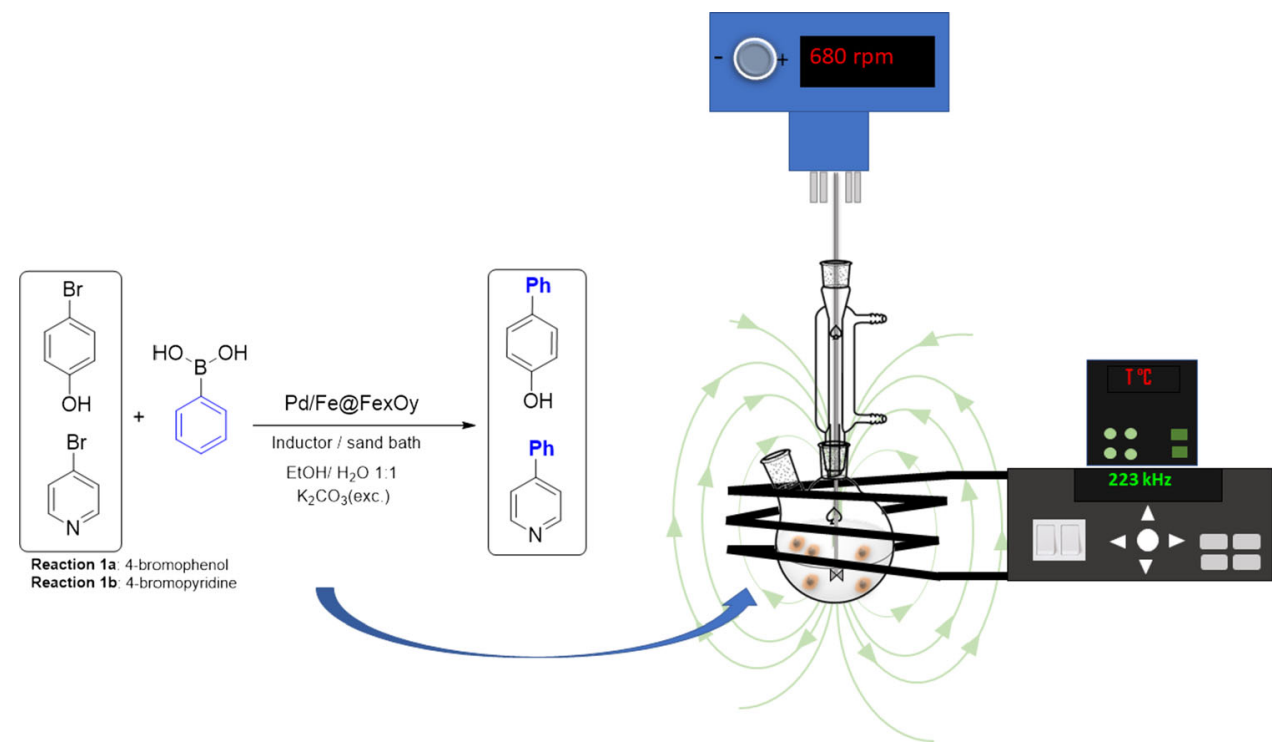

4-bromopyridine was added as hydrochloride) was dissolved in $5 \mathrm{~mL}$ of DI water and added to the mixture with $30 \mathrm{mg}$ of $\mathrm{Pd} / \mathrm{Fe} @ \mathrm{Fe}_{\mathrm{x}} \mathrm{O}_{\mathrm{y}}(5 \mathrm{~mol} \% \mathrm{Pd})$. The reaction mixture was treated as described in reaction 1a.

The conversions of the reactions were determined over time by comparing the concentrations of the final products through ${ }^{1} \mathrm{H}-\mathrm{NMR}$ analysis at the start of the reactions and after different reaction times. Some of these ${ }^{1} \mathrm{H}-\mathrm{NMR}$ spectra are provide in the Supplementary Information.

Magnetic induction heating was performed using an electromagnetic coil of an EASYHEAT LI 8310 apparatus (Ambrell) equipped with a water-cooling system. The fourth-turn coil diameter was $7.5 \mathrm{~cm}$ with a depth of $4.5 \mathrm{~cm}$. Alternating currents between 200 and 550 A were applied at a constant frequency of $223 \mathrm{kHz}$ to reach the desired temperature in the reaction medium (see Supplementary Information). A temperature signal was supplied to a temperature controller (Yokogawa) that could regulate the intensity of the alternating current flowing through the magnetic inductor coil to maintain the reaction at a constant temperature. The reaction temperature was measured using an optical fiber.

\section{Results and discussion}

\section{Catalyst characterization}

To obtain the Pd catalyst, an $\mathrm{Fe} @ \mathrm{Fe}_{3} \mathrm{O}_{4}$ solid support was fabricated, which acted as a reducing agent for the $\mathrm{Pd}^{2+}$ solution and resulted in its attachment to the surface. Figure 2 shows a characteristic X-ray diffractogram of the $\mathrm{Fe} @ \mathrm{Fe}_{3} \mathrm{O}_{4}$ precursor. All diffraction peaks match the standard JCPDS no. 01-087-0722, 01-089-2355 and 01-075-1594, which correspond to $\mathrm{Fe}, \mathrm{Fe}_{3} \mathrm{O}_{4}$, and $\mathrm{FeOOH}$, respectively. Figure 2 shows the positions of the Bragg peaks of the phases present as well as the difference between the observed and calculated patterns. Semiquantitative XRD analysis revealed the formation of $42 \mathrm{wt} . \%$ zerovalent $\mathrm{Fe}, 37$ wt. $\% \mathrm{Fe}_{3} \mathrm{O}_{4}$, and 21 wt. $\% \mathrm{FeOOH}$.

Transmission electron microscopy (TEM) analysis of $\mathrm{Fe} @ \mathrm{Fe}_{3} \mathrm{O}_{4}$ (Fig. 3a) shows the formation of two types of nanoparticles. The larger particles have sizes of 20-100 nm and correspond to $\mathrm{Fe}$ and $\mathrm{Fe}_{3} \mathrm{O}_{4}$; these particles have a cuboid appearance and form aggregated, cross-linked chains owing to their magnetic nature (Figure S1, Supplementary Information) [30]. The smaller particles (with sizes less than $10 \mathrm{~nm}$ ) would correspond to $\mathrm{Fe}(0)$ and are distributed over the surface of the $\mathrm{Fe} @ \mathrm{Fe}_{3} \mathrm{O}_{4}$ (indicated by arrows in Fig. 3a). XPS was used to analyze the surface of the material in detail and corroborate this hypothesis, as the previously reported results indicate the formation of an amorphous iron core and a shell formed mostly by $\mathrm{Fe}_{3} \mathrm{O}_{4}$ and $\mathrm{FeOOH}[31,36]$. Figure $3 \mathrm{~b}$ shows the $\mathrm{Fe}$ 
Figure $2 \mathrm{XRD}$ diffractogram calculated, and difference patterns are shown.

Semiquantitative XRD analysis and the Bragg positions of the phases present are included. of $\mathrm{Fe} @ \mathrm{Fe}_{3} \mathrm{O}_{4}$. The observed,

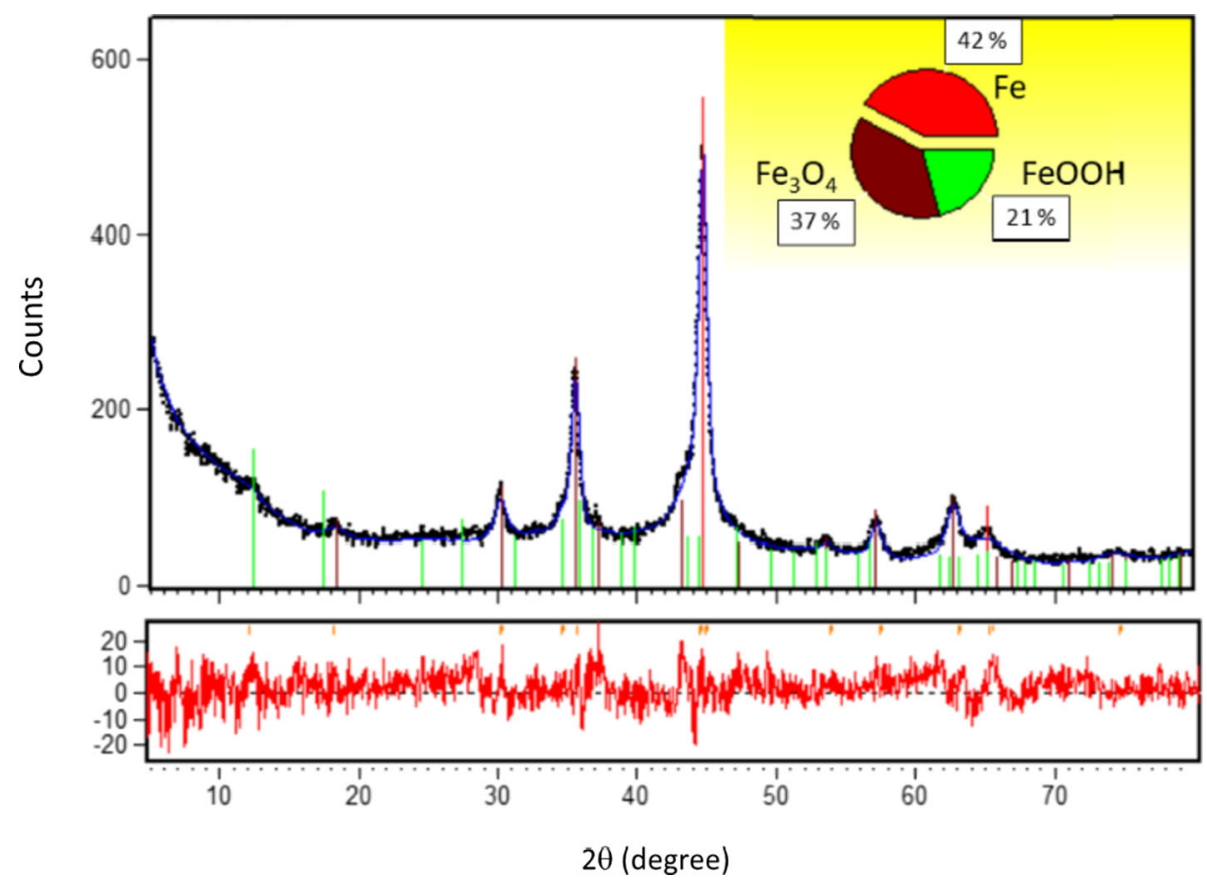

Figure 3 a TEM micrograph and $\mathbf{b}$ Fe $2 p$ XPS (Al-K $\alpha)$ spectrum of $\mathrm{Fe} @ \mathrm{Fe}_{3} \mathrm{O}_{4}$.
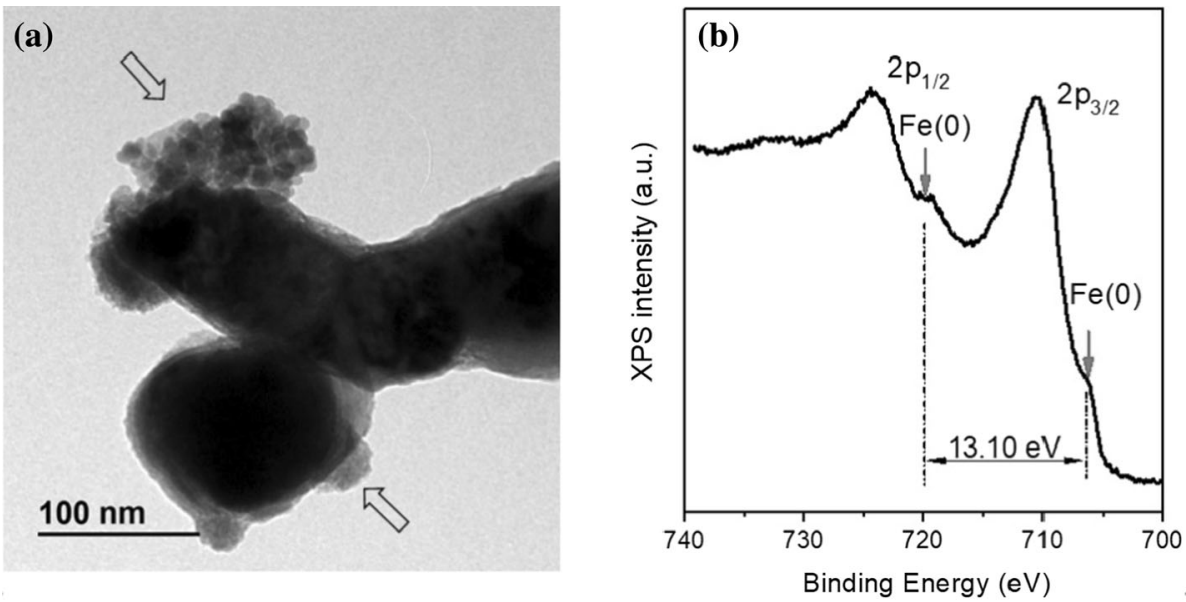

$2 \mathrm{p}$ core-level emission corresponding to the $\mathrm{Fe} @ \mathrm{Fe}_{3}$ $\mathrm{O}_{4}$ sample. The XPS spectrum shows the photoelectron emission of the complex lineshape mainly dominated by two wide peaks corresponding to the spin-orbit $3 / 2$ and $1 / 2$ doublet. The oxidation states can be determined by comparing the binding energy positions of both peaks, their energy splitting, and the presence/absence of characteristic satellites, in comparison with pure iron and standard iron oxide compounds [38, 39]. In our case, the analysis of the multiplet structure only reveals a very weak emission above the $2 \mathrm{p}_{1 / 2}$ component and a negligible signal between the doublet; it is noteworthy that the emission at lower binding energy comes from $\mathrm{Fe}(0)$, as discussed below. In accordance with this result, together with the binding energy values of $2 \mathrm{p}_{3 / 2}$ $(710.2 \mathrm{eV})$ and splitting (13.6 eV), we suggest that the surface oxide signal arises mainly from $\mathrm{Fe}_{3} \mathrm{O}_{4}$. In addition, from the detailed analysis of the spectral lineshape, a prominent emission at $706.7 \mathrm{eV}$ (arrow on the right in the figure) significantly shifted to a lower binding energy than the oxide can be observed. According to this binding energy value, the signal can be attributed to $2 \mathrm{p}_{3 / 2}$ from zerovalent $\mathrm{Fe}$, which also has its spin-orbit counterpart (arrow on the left of the figure) shifted by $13.10 \mathrm{eV}$. Therefore, the presence of both components and the characteristic splitting provides a straightforward confirmation of 
Figure 4 XRD diffractogram of $\mathrm{Pd} / \mathrm{Fe} @ \mathrm{Fe}_{3} \mathrm{O}_{4}$. The observed (dots), calculated (blue line) and difference patterns are shown.

Semiquantitative XRD analysis and the Bragg positions of the phases present are included.

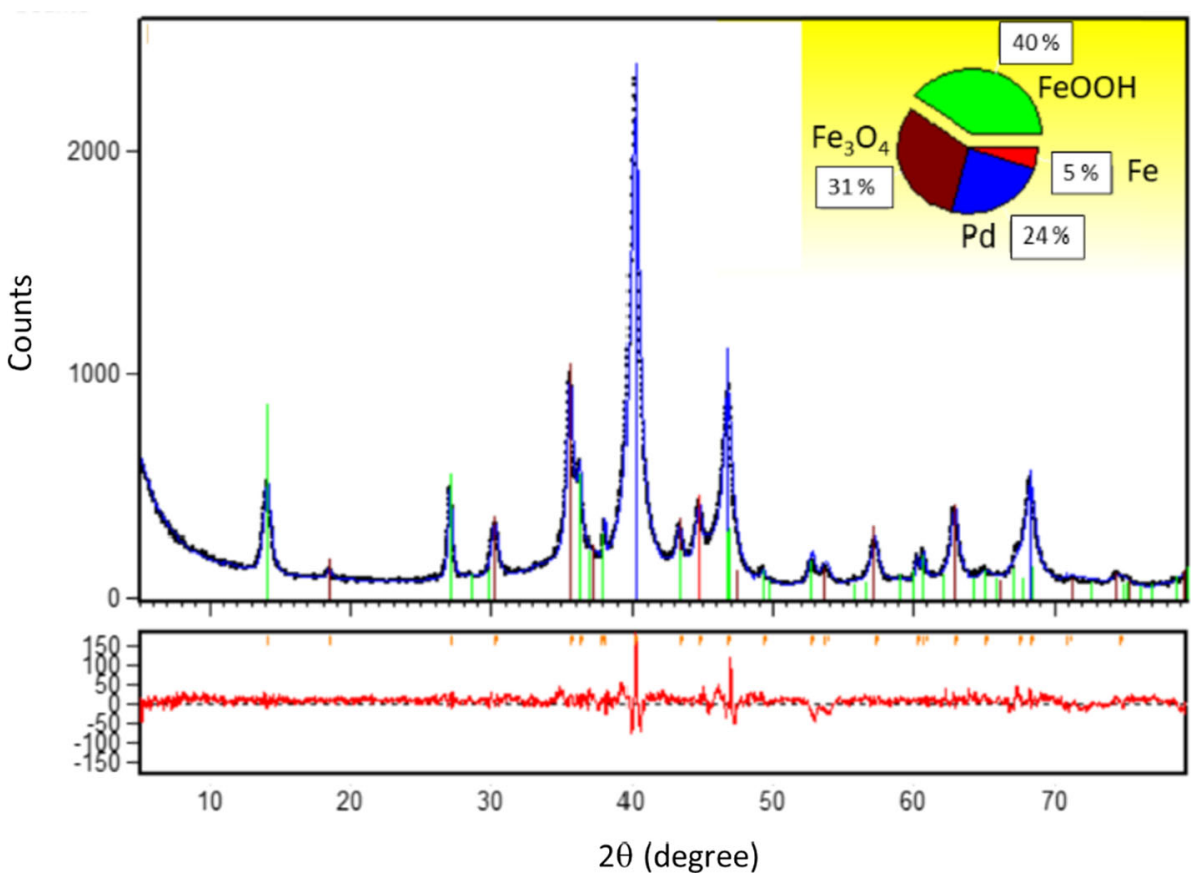

the existence of a significant amount of $\mathrm{Fe}(0)$ in the sample surface.

$\mathrm{Fe} @ \mathrm{Fe}_{3} \mathrm{O}_{4}$ can act as a reducing agent, which adsorbs and reduces $\mathrm{Pd}^{2+}$ to zerovalent Pd during the synthesis process. Figure 4 shows the diffractogram obtained for the $\mathrm{Pd} / \mathrm{Fe} @ \mathrm{Fe}_{3} \mathrm{O}_{4}$ sample, in which peaks corresponding to $\mathrm{Fe}, \mathrm{Fe}_{3} \mathrm{O}_{4}, \mathrm{FeOOH}$, and Pd (JCPDS no. 03-065-6174) are observed, demonstrating the successful anchoring of $\mathrm{Pd}$ to the substrate. Semiquantitative XRD analysis indicated that the sample contained $24 \mathrm{wt} . \% \mathrm{Pd}, 5 \mathrm{wt} . \% \mathrm{Fe}, 40$ wt. $\% \mathrm{FeOOH}$, and 31 wt. $\% \mathrm{Fe}_{3} \mathrm{O}_{4}$. The amount of $\mathrm{Pd}$ determined through semiquantitative analysis corresponded with ICP-OES results obtained for the digested $\mathrm{Pd} / \mathrm{Fe} @ \mathrm{Fe}_{3} \mathrm{O}_{4}$ sample (35 $\pm 1 \mathrm{wt}$ \% Pd).

The $\mathrm{Pd} / \mathrm{Fe} @ \mathrm{Fe}_{3} \mathrm{O}_{4}$ TEM image (Figure S2) shows an increase in needle-like structures that are characteristic of $\mathrm{FeOOH}$ [40]. In addition, larger particles, probably corresponding to $\mathrm{Fe}_{3} \mathrm{O}_{4}$, and darker particles of Pd and Fe are also shown.

Figure $5 \mathrm{a}$ displays the normalized $\mathrm{Fe} 2 \mathrm{p}$ spectra of $\mathrm{Fe} @ \mathrm{Fe}_{3} \mathrm{O}_{4}$ and $\mathrm{Pd} / \mathrm{Fe} @ \mathrm{Fe}_{3} \mathrm{O}_{4}$. Upon comparison, important lineshape differences can be observed clearly. First, the characteristic emission of $\mathrm{Fe}(0)$ is missing on the surface of the $\mathrm{Pd} / \mathrm{Fe} @ \mathrm{Fe}_{3} \mathrm{O}_{4}$ sample. Second, there is a shift to higher binding energy values for both $2 p_{3 / 2}$ and $2 p_{1 / 2}$ oxide peaks, which is
Figure 5 XPS (Al-K $\alpha)$ spectra: a normalized $\mathrm{Fe} 2 \mathrm{p}$ spectra of $\mathrm{Fe} @ \mathrm{Fe}_{3} \mathrm{O}_{4}$ and $\mathrm{Pd} /$ $\mathrm{Fe} @ \mathrm{Fe}_{3} \mathrm{O}_{4}$ b Pd 3d core level of $\mathrm{Pd} / \mathrm{Fe} @ \mathrm{Fe}_{3} \mathrm{O}_{4}$.
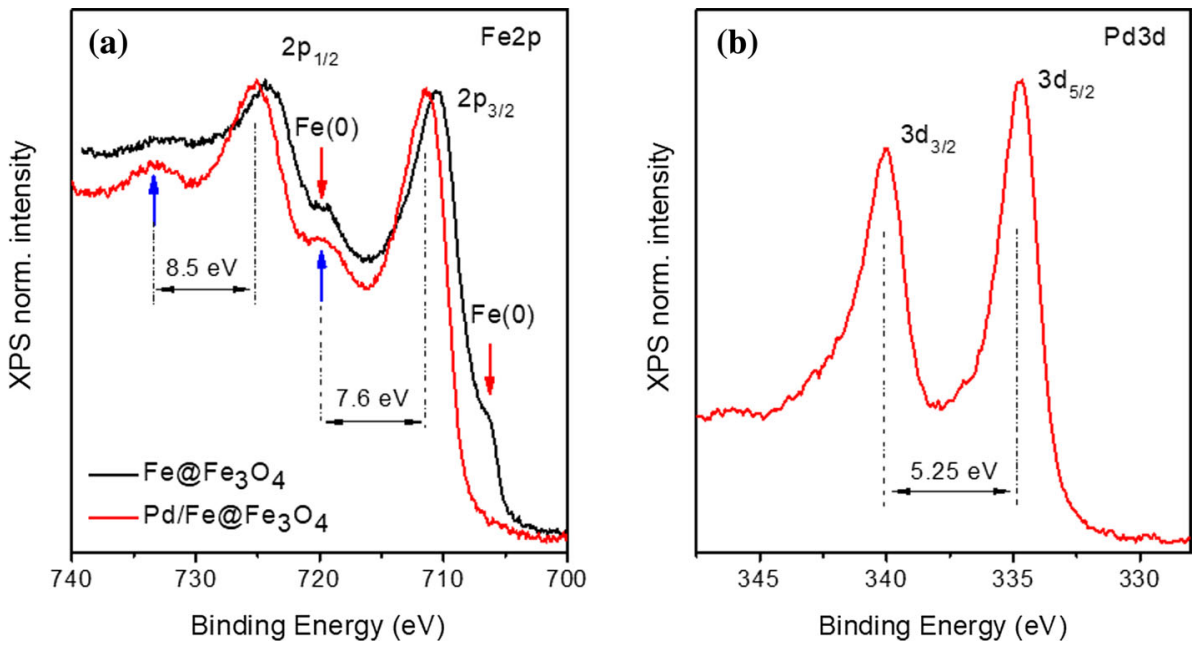
tentatively indicative of $\mathrm{Fe}^{3+}$ enrichment. In addition, a multiplet structure appears at higher energy values for each doublet peak. Following a similar analysis to that of Fig. 3b, the separation between the satellites and main peaks, 7.6 and $8.5 \mathrm{eV}$, respectively, is consistent with the majority presence of $\mathrm{Fe}^{3+}$, which can be understood as additional $\mathrm{FeO}-\mathrm{OH}$ bonds present on the surface of this sample [39]. This result agrees with the increased emission of the $\mathrm{OH}$ component in the $\mathrm{O} 1 \mathrm{~s}$ core-level spectrum located at higher energy values from the signal ascribed to oxygen in the metallic oxide lattice (not showed). Figure $5 b$ shows the XPS data of the Pd 3d core-level of Pd/Fe@ $\mathrm{Fe}_{3} \mathrm{O}_{4}$. In contrast to the Fe species, the $\mathrm{Pd} 3 \mathrm{~d}$ spectrum is basically composed of the well-defined and narrow $3 d_{5 / 2}$ and $3 d_{5 / 2}$ peaks, located at the right binding energy of $334.8 \mathrm{eV}$ and with an energy splitting of $5.25 \mathrm{eV}$, ascribed to $\mathrm{Pd}(0)$ [36]. Note that there exists a subtle emission on the higher-energy side of the doublet owing to the residual formation of native oxide due to air exposure of the sample prior to XPS measurements. Therefore, a chemical reduction from $\mathrm{Pd}^{2+}$ to $\mathrm{Pd}(0)$ occurred during the synthesis of $\mathrm{Pd} /$ $\mathrm{Fe} @ \mathrm{Fe}_{3} \mathrm{O}_{4}$.

The Mössbauer spectra of the catalyst and support, obtained at different stages of the synthetic process, are shown in Fig. 6. The $\mathrm{Fe}_{0} \mathrm{Fe}_{3} \mathrm{O}_{4}$ spectrum was fitted by overlapping a central doublet (with $\delta=0.36 \mathrm{~mm} / \mathrm{s}$ and $\Delta E_{Q}=0.7 \mathrm{~mm} / \mathrm{s}$ ) and a sextet (with $\delta=0.02 \mathrm{~mm} / \mathrm{s}, \quad \Delta E_{Q}=0.0 \mathrm{~mm} / \mathrm{s} \quad$ and $H=33.1 \mathrm{~T})$, corresponding to $\mathrm{FeOOH}$ and $\mathrm{Fe}(0)$ detected by $\mathrm{XRD}$, respectively. In addition, a broad sextet, corresponding to the $\mathrm{Fe}_{3} \mathrm{O}_{4}$ phase according to the $\mathrm{XRD}$ results, was also present. This subspectrum was fitted as a hyperfine field distribution (inset in Fig. 6a) that reflects the randomness of the environment of the Fe atoms owing to the wide size distribution of the nanoparticles or poor crystallinity. The most probable hyperfine magnetic field value is $25.2 \mathrm{~T}$, which is much lower than values corresponding to bulk-sized magnetite. The spectrum of bulk-sized magnetite is formed by two sextets, of 45 and $49 \mathrm{~T}$, which correspond to $\mathrm{Fe}$ in the tetrahedral and octahedral sites of the spinel lattice, respectively. The relative areas of the subspectra in the $\mathrm{Fe} @ \mathrm{Fe}_{3} \mathrm{O}_{4}$ sample were $31 \% \mathrm{Fe}(0), 16 \% \mathrm{FeOOH}$, and $53 \% \mathrm{Fe}_{3} \mathrm{O}_{4}$. Since the area of each Mössbauer subspectrum can be related to the population of $\mathrm{Fe}$ atoms, assuming that the Lamb-Mössbauer factor is identical for all sites and compounds, the sample composition determined by Mössbauer spectroscopy coincides with that semiquantitatively determined by $\mathrm{X}$-ray diffraction. The spectrum of $\mathrm{Pd} / \mathrm{Fe} @ \mathrm{Fe}_{3} \mathrm{O}_{4}$ (Fig. 6b) is formed by the $\mathrm{FeOOH}$ doublet, $\mathrm{Fe}(0)$ sextet, and two additional sextets with $H=43.0 \mathrm{~T}$ and $48.4 \mathrm{~T}$ that correspond to magnetite. The transformation of the magnetic field distribution in the $\mathrm{Fe} @ \mathrm{Fe}_{3} \mathrm{O}_{4}$ spectrum into the two well-defined sextets characteristic of magnetite indicates that the increase in temperature due to $\mathrm{Pd}^{2+}$ reduction, which occurs during sonication of the solution, is sufficient for the growth of nanoparticles. In contrast, a decrease from 31 to $11 \%$ in the area of the $\mathrm{Fe}(0)$ subspectrum and an increase from 16 to $33 \%$ in the area of the $\mathrm{FeOOH}$ subspectrum are observed. Therefore, it can be concluded that under these reaction conditions, $\mathrm{Fe}(0)$ is mainly oxidized to $\mathrm{FeOOH}$. The mechanism of incorporation of Pd into the catalyst could be explained by the reduction of $\mathrm{Pd}^{2+}$ with $\mathrm{Fe}(0)$ on the surface, as well as by the coordination of the metal in solution with the exposed hydroxyl groups on the particle surface, followed by reduction by the $\mathrm{Fe}(0)$ that contains the core. Both processes can occur simultaneously.
Figure 6 Mössbauer spectra of $\mathbf{a} \mathrm{Fe} @ \mathrm{Fe}_{3} \mathrm{O}_{4}$ and b $\mathrm{Pd} /$ $\mathrm{Fe} @ \mathrm{Fe}_{3} \mathrm{O}_{4}$. (a)

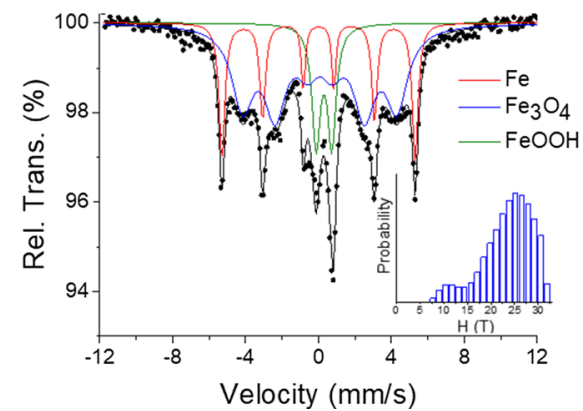

(b)

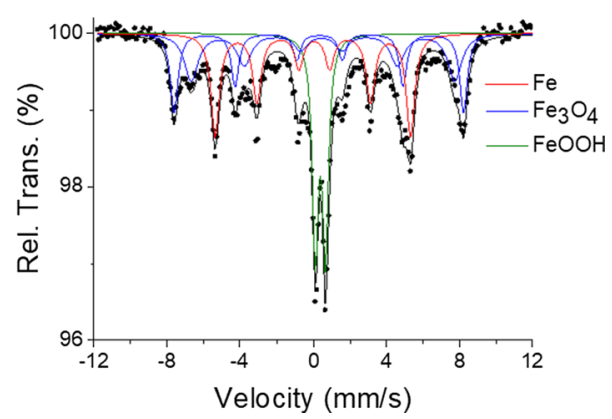


Table 1 Conversion (\%) of Suzuki-Miyaura coupling reactions

Reaction time (h)

\section{Suzuki-Miyaura coupling reactions}

The Suzuki-Miyaura reaction was performed as a model to compare the efficiency obtained by thermalbath heating and heating by magnetic induction. The temperature of the reaction solution was considered as the reaction temperature (see Figure S3).

To optimize the reaction time required for maximum conversion, aliquots of the mixture taken from the reaction of phenylboronic acid with 4-bromophenol at $50{ }^{\circ} \mathrm{C}$ were analyzed at 2,4 , and $6 \mathrm{~h}$ (Table 1). The results indicate that for both heating systems, there is a slight increase in the conversion with an increase in the reaction time from 2 to $4 \mathrm{~h}$; however, after $4 \mathrm{~h}$, a further increase in the reaction time to $6 \mathrm{~h}$ resulted in slight fluctuations in conversion, rather than progress. Based on these results, reactions 1a-b were conducted over $4 \mathrm{~h}$. The experiments were carried out three times, and the data are presented as the average of the independent reactions.

Table 1 shows the conversions of the coupling reactions between phenylboronic acid and 4-bromophenol and between phenylboronic acid and 4-bromopyridine as a function of the reaction temperature, using both heating methods.

The data indicate that heating by means of a magnetic inductor results in higher conversions for both reactions compared to that achieved using thermal bath. An increase in the temperature results in a slight increase in the substrate conversion under thermal-bath and induction heating conditions. On average, for the reaction between phenylboronic acid and 4-bromophenol, 25-30\% higher conversions were observed with magnetic induction heating compared to thermal-bath heating. Although the solution temperature was identical, induction produced a higher temperature on the surfaces of the nanoparticles. We can assume that the temperature on the nanoparticle surfaces increases by more than $40{ }^{\circ} \mathrm{C}$ by comparing the conversion achieved at $70{ }^{\circ} \mathrm{C}$ using a thermal bath with that achieved at $30{ }^{\circ} \mathrm{C}$ with magnetic induction heating. It has been demonstrated that the local heating of MNPs can generate a gradient of $45^{\circ} \mathrm{C}$ at a distance of $0.5 \mathrm{~nm}$ from the surface of the MNPs in order to decay exponentially with distance [41]. Published studies suggest that reactions catalyzed by solid-supported $\mathrm{Pd}$ occur in solution owing to 
leaching and subsequent redeposition of $\mathrm{Pd}$ [3]. Although we may assume that because of the increased temperature on the surface of the nanoparticles, where Pd is located, the reaction could occur superficially, we cannot rule out the possibility of some dissolution of $\mathrm{Pd}$ in the medium and subsequent redeposition. The temperature varies at a certain distance from the nanoparticles, but this decay is insignificant at a short distance. More precise experiments on the variation of temperature from the nanoparticles to the medium and the gradient in the concentration of $\mathrm{Pd}$ could provide insight into the mechanism. Regardless of the mechanism taking place, it is evident that the catalytic reaction occurs close enough to the particle surface for local heating to accelerate the reaction rate owing to the increase in temperature relative to the bulk solution. Although the reaction is temperature dependent, when the surfaces of the nanoparticles reach a certain temperature, an increase in the reaction-medium temperature does not considerably improve the conversion efficiency.

A relatively high conversion was achieved for the Suzuki-Miyaura coupling of phenylboronic acid and bromophenol, even at $30^{\circ} \mathrm{C}$, but the reaction with 4-bromopyridine requires a higher temperature; therefore, it does not occur at $30^{\circ} \mathrm{C}$ in a thermal bath, and the yield obtained by magnetic induction heating is only $6 \%$ on average (Table 1 ). The conversions obtained at 70 and $80^{\circ} \mathrm{C}$ for the reaction between phenylboronic acid and 4-bromopyridine heated by magnetic induction were $50 \%$ higher than those obtained at the same temperatures in a thermal bath (Table 1).

The heterogeneous $\mathrm{Pd} / \mathrm{Fe} @ \mathrm{Fe}_{3} \mathrm{O}_{4}$ catalyst proved to be efficient in the Suzuki-Miyaura reactions

Table 2 Conversion achieved for the reaction between 4-bromophenol and phenylboronic acid performed during $4 \mathrm{~h}$ at $50{ }^{\circ} \mathrm{C}$ under magnetic field induction $(223 \mathrm{kHz})$

\begin{tabular}{lll}
\hline Reactant & Cycle & Conversion (\%) \\
\hline \multirow{2}{*}{$*$} & 1 & 81 \\
${ }^{*} \mathrm{H}-\mathrm{NMR}$ conversions as average of 2 independent reactions with \\
$8 \%$ deviation
\end{tabular}

studied, especially under magnetic induction heating. Subsequently, the reusability of the catalysts was assessed. Table 2 shows the evolution of the efficiency of the catalyst over three Suzuki-Miyaura coupling reaction cycles between phenylboronic acid and 4-bromophenol at $50{ }^{\circ} \mathrm{C}$. Between each reaction cycle, the catalyst was recovered with a magnet, washed with distilled water and ethanol several times, and dried under vacuum.

To determine the reason for the decrease in the reactivity of the catalyst in successive cycles, XPS measurements of the samples were carried out after reuse. Figure $7 \mathrm{a}, \mathrm{b}$ compares the XPS spectra of $\mathrm{Pd} 3 \mathrm{~d}$ and $\mathrm{C} 1 \mathrm{~s}$ corresponding to $\mathrm{Pd} / \mathrm{Fe} @ \mathrm{Fe}_{3} \mathrm{O}_{4}$ fresh and reused samples. Note that the detailed XPS lineshape analysis reveals no significant differences between $\mathrm{Fe}$ $2 \mathrm{p}$ (not shown but similar to that displayed in Fig. 5a) and $\mathrm{Pd} 3 \mathrm{~d}$ spectra in both samples. In addition, the Pd 3d spectrum includes more prominent asymmetric peaks with a minor contribution of surface native oxide, most likely because the surface of the nanoparticles is covered with a higher amount of $C$ (see below), which reduces their exposure to air after preparation. The only difference between the spectra is the absolute emission intensity, in which signals are attenuated by the higher amount of $C$ present on the surface. However, this modification did not change the relative surface atomic concentration ratio, suggesting low leaching of Pd. ICP-OES analysis of the catalyst revealed a slight variation in the composition of the MNPs with increased use; the Fe/ Pd ratio changed by less than $5 \%$ after each cycle. In contrast, there exists a significant increase in the C concentration on the surface, which might be responsible of the poison effect observed in the catalytic behavior of the samples after use.

The loss in catalyst efficiency observed for Pd/ $\mathrm{Fe} @ \mathrm{Fe}_{3} \mathrm{O}_{4}$ over three cycles is slightly greater than that reported for catalysts consisting of Pd supported on coated $\mathrm{Fe}_{3} \mathrm{O}_{4}$, such as $\mathrm{Fe}_{3} \mathrm{O}_{4} / \mathrm{PEG} / \mathrm{Pd}$ [35] or $\mathrm{Fe}_{3} \mathrm{O}_{4} / \mathrm{DA}-\mathrm{Pd}$ [22]. However, the use of uncoated magnetite offers the advantage of a facile and economical synthetic process.

\section{Conclusions}

We have shown that it is possible to perform SuzukiMiyaura reactions under magnetic induction heating using Pd-functionalized MNPs without the addition 
Figure $7 \mathrm{XPS}(\mathrm{Al}-\mathrm{K} \alpha)$ spectra of $\mathrm{Pd} / \mathrm{Fe} @ \mathrm{Fe}_{3} \mathrm{O}_{4}$ fresh and reused. a Pd $3 \mathrm{~d}$ core level, b $\mathrm{C} 1 \mathrm{~s}$ core level of the catalyst.
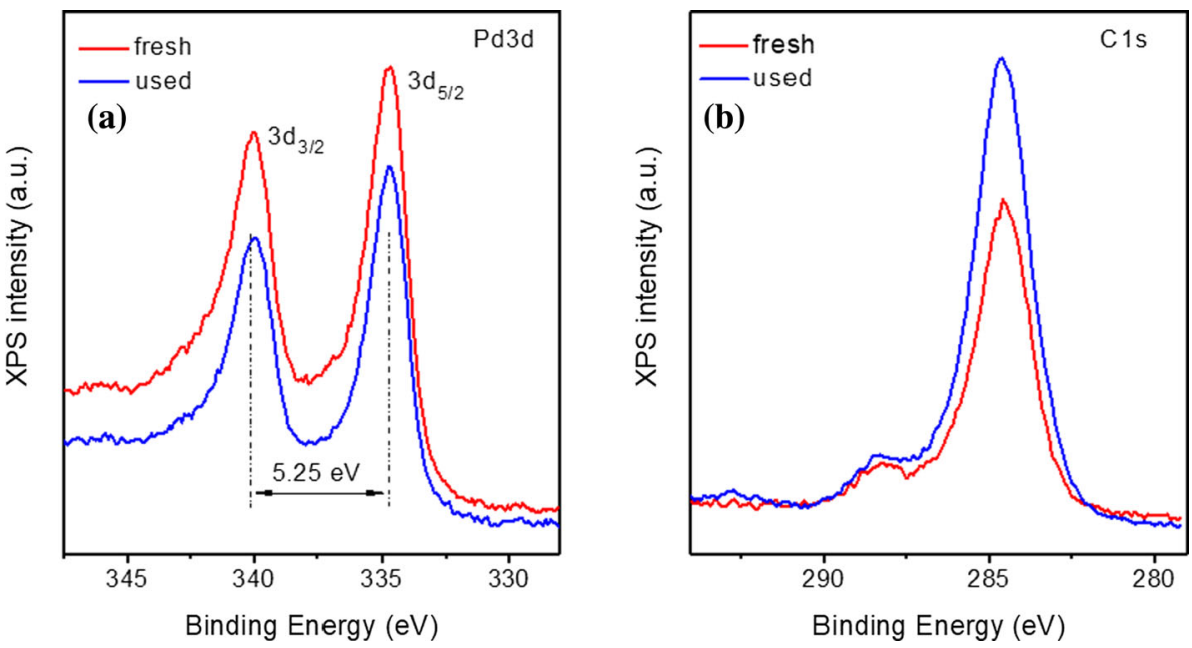

of an organic coating, which saves time and resources and makes the process more sustainable.

Magnetic induction increases the efficiency of the catalytic process by approximately $25-35 \%$ compared to the reaction in which conventional heating was applied; this is due to the temperature on the surface of the MNPs being higher than that of the solvent. This allows the reaction to proceed without the need to heat the solvent to the required temperature. Furthermore, owing to the magnetic properties of the nanoparticles, the catalyst can be easily recovered and reused. However, further studies are required to increase the number of reaction cycles in which the recovered catalyst can be used.

\section{Acknowledgements}

We are grateful for financial support provided by the Government of Spain through project PGC2018095642-B-I00. In addition, A. Villacampa and L. Duque acknowledge the Community of Madrid for Predoctoral contracts PEJD-2019-PRE/IND-15356 and PEJ-2019-AI/IND-12506, respectively, co-financed by the European Social Fund through the Youth Employment Operational Program and the Youth Employment Initiative (YEI).

\section{Funding}

Open Access funding provided thanks to the CRUECSIC agreement with Springer Nature.

\section{Declarations}

Conflict of interest The authors declare no conflict of interest.

Supplementary Information: The online version contains supplementary material available at http s://doi.org/10.1007/s10853-021-06591-w.

Open Access This article is licensed under a Creative Commons Attribution 4.0 International License, which permits use, sharing, adaptation, distribution and reproduction in any medium or format, as long as you give appropriate credit to the original author(s) and the source, provide a link to the Creative Commons licence, and indicate if changes were made. The images or other third party material in this article are included in the article's Creative Commons licence, unless indicated otherwise in a credit line to the material. If material is not included in the article's Creative Commons licence and your intended use is not permitted by statutory regulation or exceeds the permitted use, you will need to obtain permission directly from the copyright holder. To view a copy of this licence, visit http://creativecommons.org/licen ses $/$ by $/ 4.0 /$.

Supplementary Information: The online version contains supplementary material available at http s://doi.org/10.1007/s10853-021-06591-w. 


\section{References}

[1] Cenini S, Gallo E, Caselli A et al (2006) Coordination chemistry of organic azides and amination reactions catalyzed by transition metal complexes. Coord Chem Rev 250:1234-1253

[2] Hashmi ASK (2007) Gold-catalyzed organic reactions. Chem Rev 107:3180-3211

[3] Yin L, Liebscher J (2007) Carbon-carbon coupling reactions catalyzed by heterogeneous palladium catalysts. Chem Rev 107:133-173

[4] Kuninobu Y, Takai K (2011) Organic reactions catalyzed by rhenium carbonyl complexes. Chem Rev 111:1938-1953

[5] Hernández JG, Friščić T (2015) Metal-catalyzed organic reactions using mechanochemistry. Tetrahedron Lett 56:4253-4265

[6] Nájera C, Beletskaya IP, Yus M (2019) Metal-catalyzed regiodivergent organic reactions. Chem Soc Rev 48:4515-4618

[7] Cheng WM, Shang R (2020) Transition metal-catalyzed organic reactions under visible light: recent developments and future perspectives. ACS Catal 10:9170-9196

[8] Miyaura N, Yamada K, Suzuki A (1979) A new stereospecific cross-coupling by the palladium-catalyzed reaction of 1-alkenylboranes with 1-alkenyl or 1-alkynyl halides. Tetrahedron Lett 20:3437-3440. https://doi.org/10.1016/S0 040-4039(01)95429-2

[9] Hayler JD, Leahy DK, Simmons EM (2019) A pharmaceutical industry perspective on sustainable metal catalysis. Organometallics 38:36-46. https://doi.org/10.1021/acs.orga nomet.8b00566

[10] Das P, Bora U, Tairai A, Sharma C (2010) Triphenylphosphine chalcogenides as efficient ligands for room temperature palladium(II)-catalyzed Suzuki-Miyaura reaction. Tetrahedron Lett 51:1479-1482. https://doi.org/10.1016/j.te tlet.2010.01.032

[11] Climent MJ, Corma A, Iborra S, Mifsud M (2007) Heterogeneous palladium catalysts for a new one-pot chemical route in the synthesis of fragrances based on the heck reaction. Adv Synth Catal 349:1949-1954. https://doi.org/10.10 02/ADSC.200700026

[12] Chen Y, Wang M, Zhang L et al (2017) Poly(: O aminothiophenol)-stabilized $\mathrm{Pd}$ nanoparticles as efficient heterogenous catalysts for Suzuki cross-coupling reactions. RSC Adv 7:47104-47110. https://doi.org/10.1039/c7ra 09947a

[13] Rahimi L, Mansoori Y, Nuri A, Esquivel D (2020) A new magnetically retrievable porous supported catalyst for the Suzuki-Miyaura cross-coupling reaction. ChemistrySelect 5:11690-11697. https://doi.org/10.1002/slct.202003198
[14] Martin R, Buchwald SL (2008) Palladium-catalyzed suzukimiyaura cross-coupling reactions employing dialkylbiaryl phosphine ligands. Acc Chem Res 41:1461-1473

[15] Fihri A, Bouhrara M, Nekoueishahraki B et al (2011) Nanocatalysts for Suzuki cross-coupling reactions. Chem Soc Rev 40:5181-5203. https://doi.org/10.1039/c1cs15079k

[16] Veisi H, Amini Manesh A, Eivazi N, Faraji AR (2015) Palladium nanoparticles supported on 1,3-dicyclohexylguanidine functionalized mesoporous silica SBA-15 as highly active and reusable catalyst for the Suzuki-Miyaura cross-coupling reaction. RSC Adv 5:20098-20107. https://d oi.org/10.1039/c4ra14668a

[17] Zheng Z, Li H, Liu T, Cao R (2010) Monodisperse noble metal nanoparticles stabilized in SBA-15: synthesis, characterization and application in microwave-assisted SuzukiMiyaura coupling reaction. J Catal 270:268-274. https://doi. org/10.1016/j.jcat.2010.01.004

[18] Rohani S, Mohammadi Ziarani G, Badiei A et al (2019) Mesoporous hierarchically hollow flower-like CoAlLDH@N, S-doped Graphene@Pd nanoarchitectures for heck couplings. Catal Lett 149:2984-2993. https://doi.org/ 10.1007/s10562-019-02880-x

[19] Sokolov VI, Rakov EG, Bumagin NA, Vinogradov MG (2010) New method to prepare nanopalladium clusters immobilized on carbon nanotubes: a very efficient catalyst for forming carbon-carbon bonds and hydrogenation. Fuller Nanotub Carbon Nanostructures 18:558-563. https://doi.org/ 10.1080/1536383X.2010.488077

[20] Llabrés i Xamena FX, Abad A, Corma A, Garcia H (2007) MOFs as catalysts: activity, reusability and shape-selectivity of a Pd-containing MOF. J Catal 250:294-298. https://doi. org/10.1016/j.jcat.2007.06.004

[21] Augustyniak AW, Zawartka W, Navarro JAR, Trzeciak AM (2016) Palladium nanoparticles supported on a nickel pyrazolate metal organic framework as a catalyst for Suzuki and carbonylative Suzuki couplings. Dalt Trans 45:13525-13531. https://doi.org/10.1039/c6dt02242a

[22] Long Y, Liang K, Niu J et al (2015) Agglomeration of Pd0 nanoparticles causing different catalytic activities of Suzuki carbonylative cross-coupling reactions catalyzed by PdII and Pd0 immobilized on dopamine-functionalized magnetite nanoparticles. New J Chem 39:2988-2996. https://oi.org/ 10.1039/c4nj02285h

[23] Bobb JA, Ibrahim AA, El-Shall MS (2018) Laser synthesis of carbonaceous $\mathrm{TiO}_{2}$ from metal-organic frameworks: optimum support for Pd nanoparticles for C-C cross-coupling reactions. ACS Appl Nano Mater 1:4852-4862. http s://doi.org/10.1021/acsanm.8b01045

[24] Yoon TJ, Lee W, Oh YS, Lee JK (2003) Magnetic nanoparticles as a catalyst vehicle for simple and easy 
recycling. New J Chem 27:227-229. https://doi.org/10.103 9/b209391j

[25] Shylesh S, Wang L, Demeshko S, Thiel WR (2010) Facile synthesis of mesoporous magnetic nanocomposites and their catalytic application in carbon-carbon coupling reactions. ChemCatChem 2:1543-1547. https://doi.org/10.1002/cctc. 201000215

[26] Ahmadi A, Sedaghat T, Motamedi H, Azadi R (2020) Anchoring of $\mathrm{Cu}$ (II)-Schiff base complex on magnetic mesoporous silica nanoparticles: catalytic efficacy in one-pot synthesis of 5-substituted-1H-tetrazoles, antibacterial activity evaluation and immobilization of $\alpha$-amylase. Appl Organomet Chem 34:e5572. https://doi.org/10.1002/aoc. 5572

[27] Rafiee E, Kahrizi M, Joshaghani M, Ghaderi-Sheikhi Abadi P (2016) New strategy by a two-component heterogeneous catalytic system composed of Pd-PVP-Fe and heteropoly acid as co-catalyst for Suzuki coupling reaction. Res Chem Intermed 42:5573-5585. https://doi.org/10.1007/s11164-01 $5-2387-5$

[28] Esmaeilpour M, Zahmatkesh S, Fahimi N, Nosratabadi M (2018) Palladium nanoparticles immobilized on EDTAmodified $\mathrm{Fe}_{3} \mathrm{O}_{4} @ \mathrm{SiO} 2$ nanospheres as an efficient and magnetically separable catalyst for Suzuki and Sonogashira cross-coupling reactions. Appl Organomet Chem 32:e4302. https://doi.org/10.1002/aoc.4302

[29] Li XQ, Zhang WX (2006) Iron nanoparticles: the core-shell structure and unique properties for $\mathrm{Ni}(\mathrm{II})$ sequestration. Langmuir 22:4638-4642. https://doi.org/10.1021/la060057k

[30] Macdonald JE, Kelly JA, Veinot JGC (2007) Iron/iron oxide nanoparticle sequestration of catalytic metal impurities from aqueous media and organic reaction products. Langmuir 23:9543-9545. https://doi.org/10.1021/la7011827

[31] Macdonald JE, Veinot JGC (2008) Removal of residual metal catalysts with iron/iron oxide nanoparticles from coordinating environments. Langmuir 24:7169-7177. http s://doi.org/10.1021/la8006734

[32] Ceylan S, Friese C, Lammel C et al (2008) Inductive heating for organic synthesis by using functionalized magnetic nanoparticles inside microreactors. Angew Chemie Int Ed 47:8950-8953. https://doi.org/10.1002/anie.200801474

[33] Meffre A, Mehdaoui B, Connord V et al (2015) Complex nano-objects displaying both magnetic and catalytic properties: a proof of concept for magnetically induced heterogeneous catalysis. Nano Lett 15:3241-3248. https://d oi.org/10.1021/acs.nanolett.5b00446

[34] Phenrat T, Kumloet I (2016) Electromagnetic induction of nanoscale zerovalent iron particles accelerates the degradation of chlorinated dense non-aqueous phase liquid: Proof of concept. Water Res 107:19-28. https://doi.org/10.1016/j.wa tres.2016.10.035

[35] De Cattelle A, Billen A, Brullot W et al (2019) Magnetically induced Suzuki and Sonogashira reaction performed using recyclable, palladium-functionalized magnetite nanoparticles. J Organomet Chem 899:120905. https://doi.org/10.10 16/j.jorganchem.2019.120905

[36] Zhou S, Johnson M, Veinot JGC (2010) Iron/iron oxide nanoparticles: a versatile support for catalytic metals and their application in Suzuki-Miyaura cross-coupling reactions. Chem Commun 46:2411-2413. https://doi.org/10.103 9/b922462a

[37] Brand RA (1987) Improving the validity of hyperfine field distributions from magnetic alloys. Nucl Instrum Methods Phys Res Sect B Beam Interact Mater Atoms 28:398-416. h ttps://doi.org/10.1016/0168-583X(87)90182-0

[38] Biesinger MC, Payne BP, Grosvenor AP et al (2011) Resolving surface chemical states in XPS analysis of first row transition metals, oxides and hydroxides: $\mathrm{Cr}, \mathrm{Mn}, \mathrm{Fe}, \mathrm{Co}$ and Ni. Appl Surf Sci 257:2717-2730. https://doi.org/10. 1016/J.APSUSC.2010.10.051

[39] Rubio-Zuazo J, Chainani A, Taguchi M et al (2018) Electronic structure of $\mathrm{FeO}, \gamma-\mathrm{Fe}_{2} \mathrm{O}_{3}$, and $\mathrm{Fe}_{3} \mathrm{O}_{4}$ epitaxial films using high-energy spectroscopies. Phys Rev B. https://doi. org/10.1103/PhysRevB.97.235148

[40] Cui H, Wang L, Shi M, Li Y (2017) Morphology and phase control of iron oxide polymorph nanoparticles. Mater Res Express 4:045006. https://doi.org/10.1088/2053-1591/ AA680D

[41] Riedinger A, Guardia P, Curcio A et al (2013) Subnanometer local temperature probing and remotely controlled drug release based on Azo-functionalized iron oxide nanoparticles. Nano Lett 13:2399-2406. https://doi.org/10.1021/ nl400188q

Publisher's Note Springer Nature remains neutral with regard to jurisdictional claims in published maps and institutional affiliations. 